\title{
Recurrent Bladder Urothelial Carcinoma
}

National Cancer Institute

\section{Source}

National Cancer Institute. Recurrent Bladder Urothelial Carcinoma. NCI Thesaurus. Code C126307.

The reemergence of bladder urothelial carcinoma after a period of remission. 